\title{
New Multi-Rod Linear Actuator for Direct-Drive, Wide Mechanical Bandpass Applications
}

\author{
Pierre-Emmanuel Cavarec, Hamid Ben Ahmed, and Bernard Multon, Member, IEEE
}

\begin{abstract}
In this paper, a new multi-rod linear actuator is presented. This actuator has been developed for high thrust density and wide mechanical bandpass applications. The multi-air-gap concept will first be illustrated and explained. Its advantages will be highlighted thanks to finite-element method optimizations. Next, the multi-rod prototype technology will be discussed. Lastly, experimental measurements for prototype forces will be provided.
\end{abstract}

Index Terms-High mechanical bandpass, linear permanentmagnet motor.

\section{INTRODUCTION}

$\mathbf{I}$ N ELECTRICAL drives, load is generally linked to the actuator by means of mechanical transmission (e.g., gears). This mechanical transmission is required in order to adapt the load to a standard motor (as regards type of movement, speed). This is particularly true in the case of low-speed linear loads. In those cases, the most common solution is to adapt a high-speed electrical motor to a ball-screw gear or a hydraulic system.

Another solution can be to directly connect the load to an adapted electrical actuator. We call these techniques the directdrive solutions. In a growing number of instances, direct drives are preferred to mechanical transmission for several reasons [1]. They enable the following:

- replacing gears (hence, cost savings);

- reducing noise;

- increasing movement control;

- widening the mechanical bandpass;

- lowering maintenance.

For automation applications, such as drive (plane drive, hard disk arm) or regulation (vibration compensation), mechanical bandpass represents the primary constraint. For both of these (drive and regulation), the system's mechanical time constant proves to be greatest (the sensor, computing and drive time constants are all generally lower). Direct drives (i.e., featuring no mechanical transmission) present two main advantages in such applications.

Paper IPCSD 03-029, presented at the 2002 Industry Applications Society Annual Meeting, Pittsburgh, PA, October 13-18, and approved for publication in the IEEE TRANSACTIONS ON INDUSTRY APPLICATIONS by the Electric Machines Committee of the IEEE Industry Applications Society. Manuscript submitted for review September 1, 2002 and released for publication April 16, 2003

P.-E. Cavarec was with LESiR-SATIE (UMR CNRS 8029), Ecole Normale Supérieure de Cachan-Brittany, 35170 Bruz, France. He is now with Harmonic Design Inc., Somfy International, Poway, CA 92064 USA (e-mail: PierreEmmanuel.CAVAREC@ somfy.com).

H. Ben Ahmed and B. Multon are with LESiR-SATIE (UMR CNRS 8029), Ecole Normale Supérieure de Cachan-Brittany, 35170 Bruz, France (e-mail benahmed@bretagne.ens-cachan.fr; multon@bretagne.ens-cachan.fr).

Digital Object Identifier 10.1109/TIA.2003.813705
- They eliminate the mechanical tolerance that causes the "backlash" phenomenon and, thus, lowers precision. Moreover, they introduce a sleep time into the feedback loop.

- From an overall perspective, the reduction level serves to increase system inertia due not only to inertia of the mechanical transmission itself, but to the actuator inertia as well.

The main difficulty for direct drives consists of reaching sufficient force density (i.e., force for a linear actuator, torque for a rotary actuator). The absence of gears imposes a considerably higher force. For electromagnetic actuators, however, the size (and, thus, cost) is linked to this force. We recall herein that, for electronic power converters, cost is not linked to force but to apparent power. For low-speed movement, direct drives are, therefore of interest once a sufficiently high force-to-volume ratio has been reached.

\section{Multi-Air-GAP LinEAR ACTUATORS}

In electromagnetic actuators, the mechanical constraints of a magnetic air gap cannot be overcome: the magnetic shear stress gets limited by means of both air-gap induction and electrical loading. In turn, air-gap induction is limited by the saturation of ferromagnetic materials and electric loading is limited by heating and/or demagnetization of any eventual magnets. With a copper conductor, the maximum attainable values of air-gap shear stress rarely exceed 10-20 N/cm². An actuator can thereby be described as a system for producing magnetic fields that interact inside an air gap where magnetic stress is being generated.

Another approach [2], conceived some 20 years ago, yet still rather undeveloped due to its conceptual complexity, consists of splitting the active zone so as to increase air-gap surfaces, thus giving rise to what are called "multi-air-gap structures." Let us see, in the particular case of linear actuators, which are the different solution.

\section{A. Various Types of Global-Coil, Multi-Air-Gap Actuators}

Splitting the active zone may be performed in two ways: the coil may be distributed (in distributed coil actuators) or kept whole (in global-coil actuators).

Distributed coil actuators may be viewed as the summation of several single-air-gap actuators. This category primarily features multi-discoid actuators.

For the global-coil actuator, either a variable reluctance (VR) actuator or permanent-magnet actuator is possible. VR actua- 


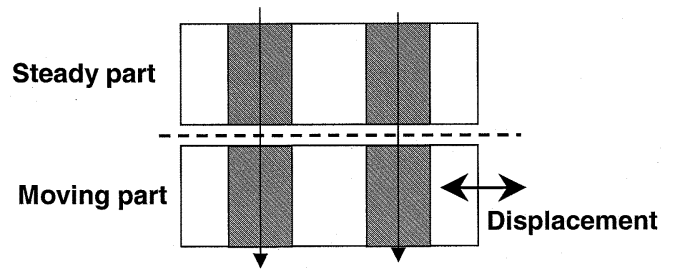

(a)

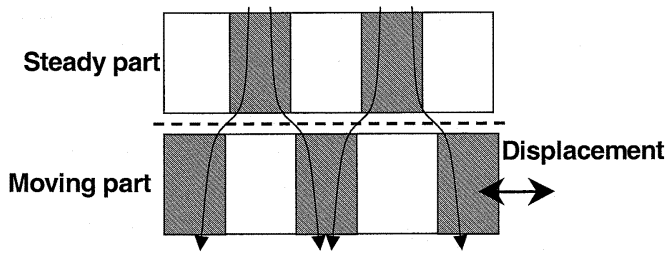

(b)

Fig. 1. Transverse flux reluctance pattern. (a) Conjunction position. (b) Opposition position.

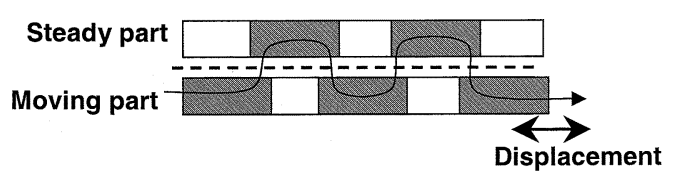

(a)

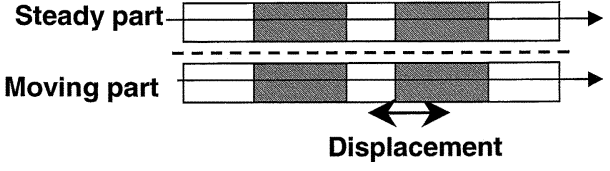

(b)

Fig. 2. Longitudinal flux reluctance pattern. (a) Conjunction position. (b) Opposition position.

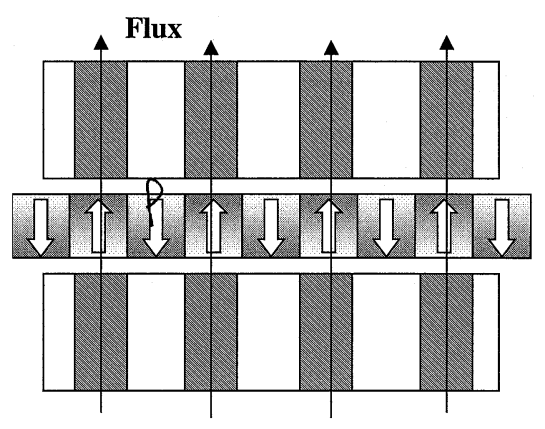

(a)

Fig. 3. (a) A-pattern, positive alignment. (b) A-pattern, negative alignment.

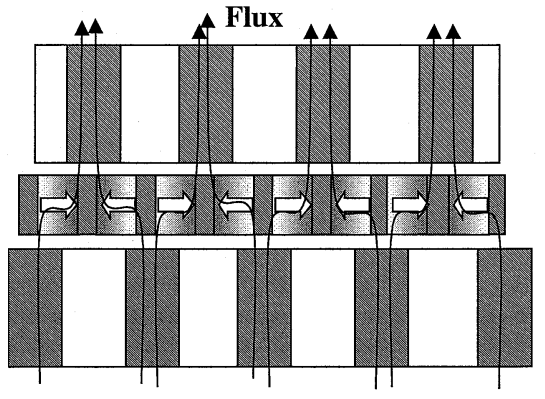

(a)

Fig. 4. (a) B-pattern, positive alignment. (b) B-pattern, negative alignment.

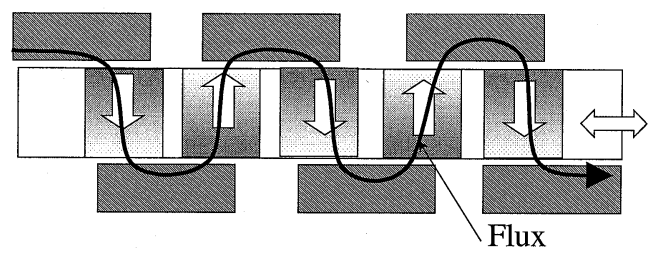

(a)

Fig. 5. (a) C-pattern, positive alignment. (b) C-pattern, negative alignment.

tors exhibit two kinds of patterns: a longitudinal flux pattern [3] (Fig. 1), where the flux goes from the top to the bottom, and a transverse flux pattern [4], where the flux goes from the left to the right (Fig. 2). In all these topologies, the return flux part is external.

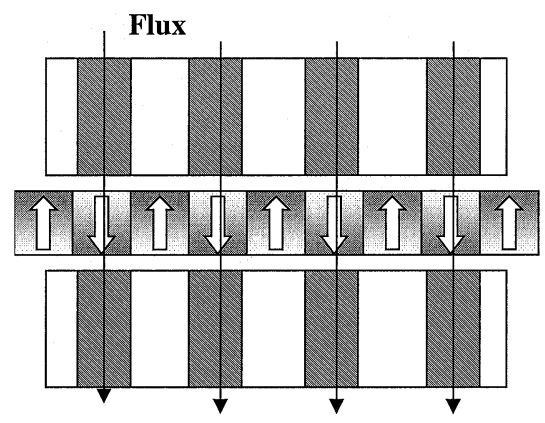

(b)

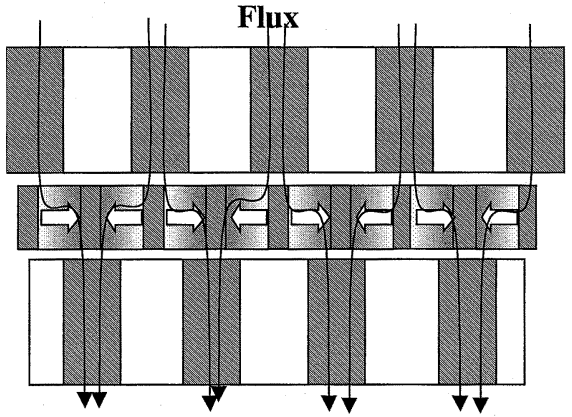

(b)

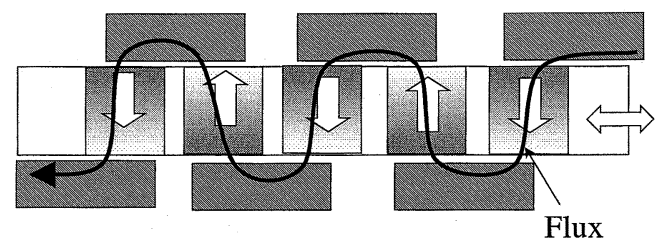

(b)

For permanent-magnet actuators, four kinds of patterns exist: longitudinal flux and magnet (A-pattern) (Fig. 3), longitudinal flux and transverse magnet (B-pattern) (Fig. 4), transverse flux and longitudinal magnet (C-pattern) (Fig. 5), and transverse flux and magnet (D-pattern) (Fig. 6). 


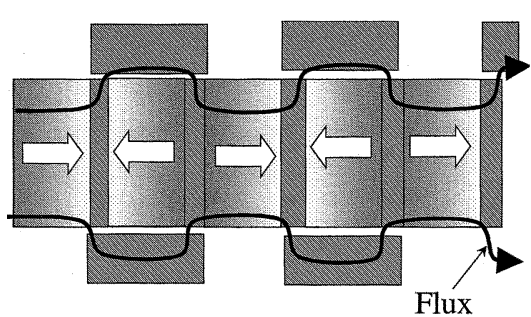

(a)

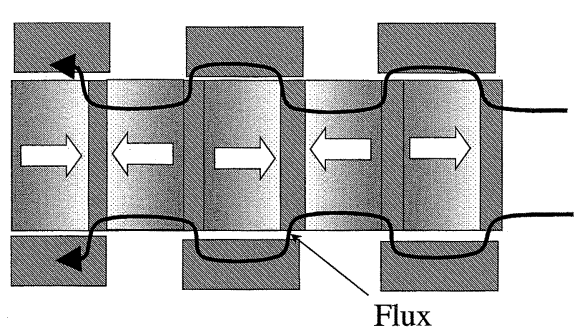

(b)

Fig. 6. (a) D-pattern, positive alignment. (b) D-pattern, negative alignment.
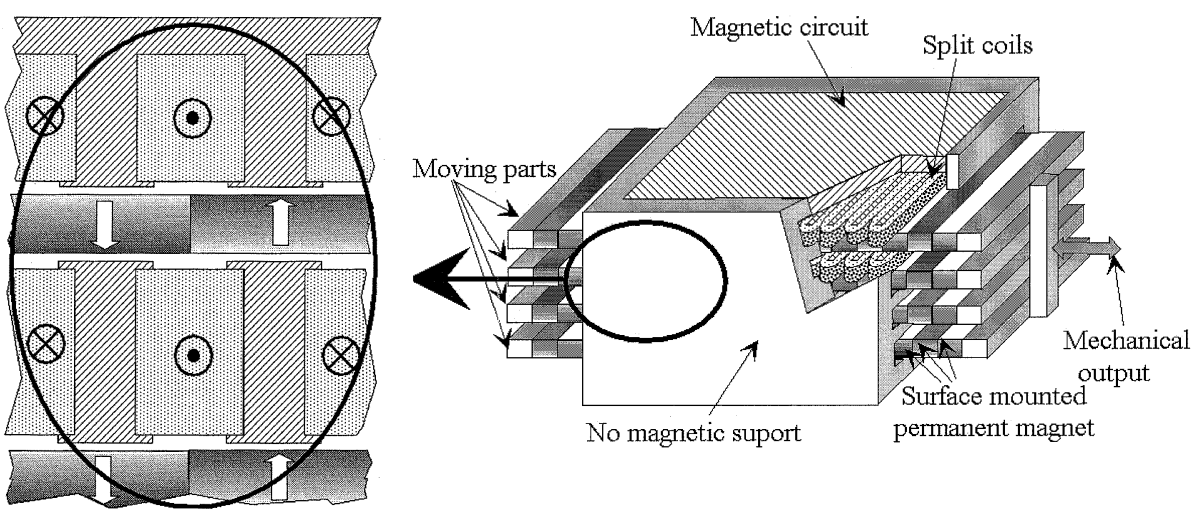

Fig. 7. Distributed coil, multi-air-gap architecture and three-dimensional (3-D) view of one phase of an actuator.
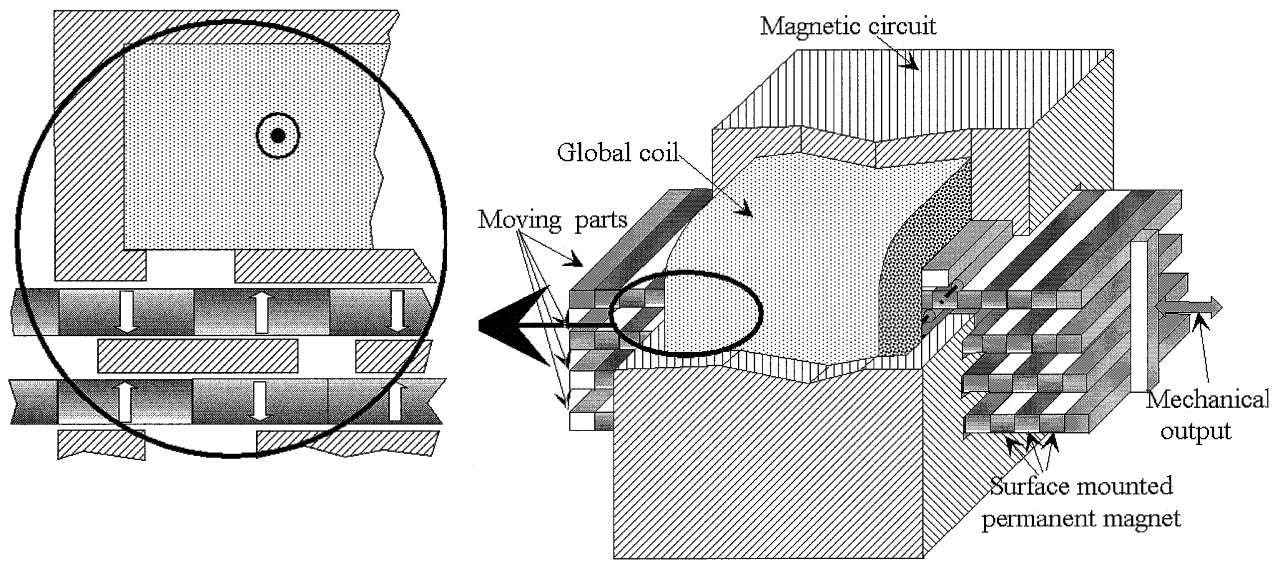

Fig. 8. Global-coil, multi-air-gap architecture and 3-D view of one phase of an actuator.

In general, the transverse flux pattern is well adapted to rotary actuators and the longitudinal flux pattern to linear actuators.

\section{B. Benefit of Global-Coil, Multi-Air-Gap Actuators}

In order to demonstrate the benefit of multi-air-gap structures, we have compared [5] two basic permanent-magnet actuator architectures [i.e., distributed coil (Fig. 7), multi-air-gap and global-coil, multi-air-gap (Fig. 8)] with the C-pattern [6].

These structures have been optimized thanks to a genetic algorithm tied to a finite-element method (FEM) (Fig. 9). The force calculation, based on the flux/magnetomotive force (MMF) [7], [8], will be presented in Section III for the multi-rod prototype.

A comparison of the force-to-volume ratio for various volumes helps summarize these results. In the present case, both the air-gap thickness $(1 \mathrm{~mm}$ ) and cooling conditions (airflow cooling on the exterior surface) are the same for the two types of actuators (Fig. 10).

It can be concluded that, under given cooling conditions, a volume always exists for which global-coil actuators outperform distributed coil actuators. This volume is linked directly to air-gap thickness: as the air gap narrows, the multi-air-gap actuators become more effective.

\section{New Multi-Rod ACtuATOR}

\section{A. General Presentation of the Multi-Rod Actuator}

The actuator presented herein is the result of our previous experimental work with multi-air-gap actuators [4], [9] [10]. Its introduction has been focused on wide bandpass applications. The mechanical setup has been designed to minimize air-gap thickness. In order to enhance mechanical precision, all of the 


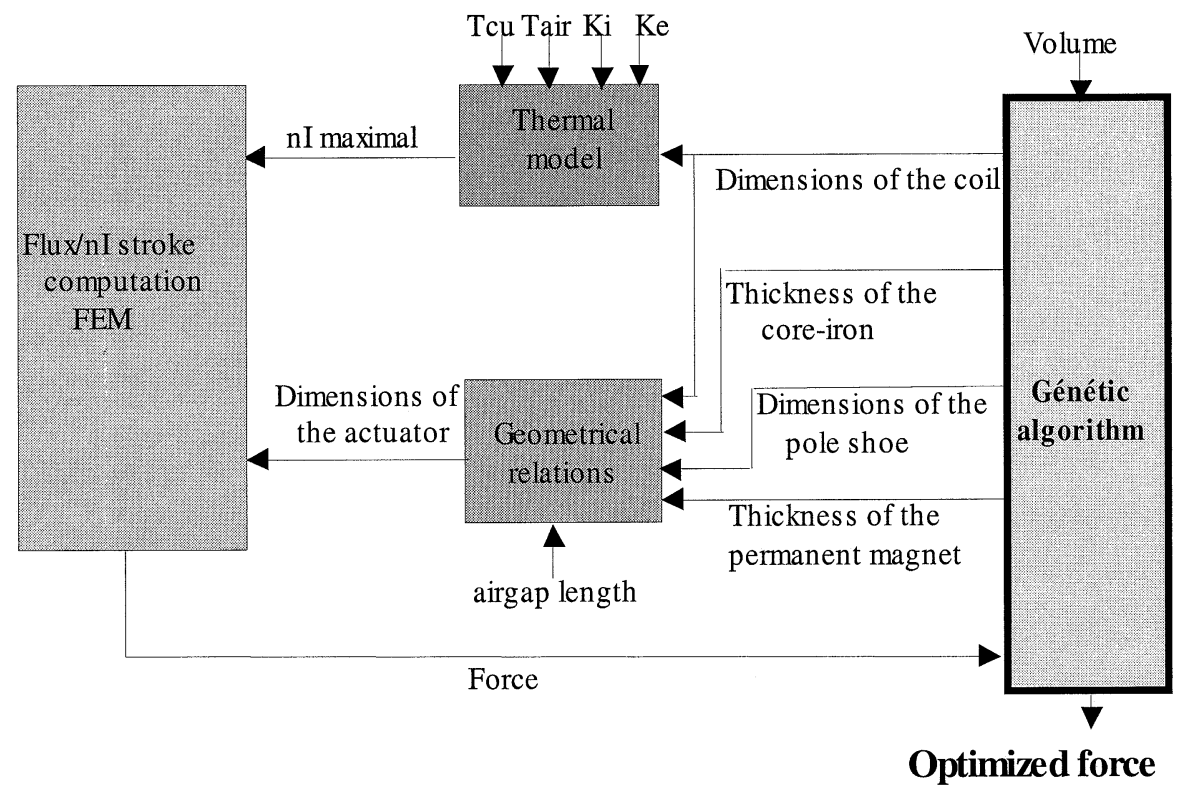

Fig. 9. Diagram of the optimization method.

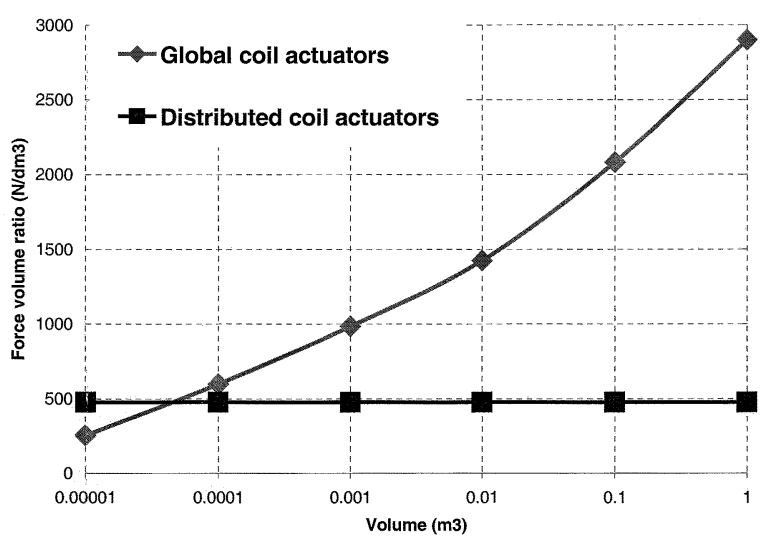

Fig. 10. Scale effect on force-to-volume ratio for optimized multi-air-gap architectures of linear actuators.

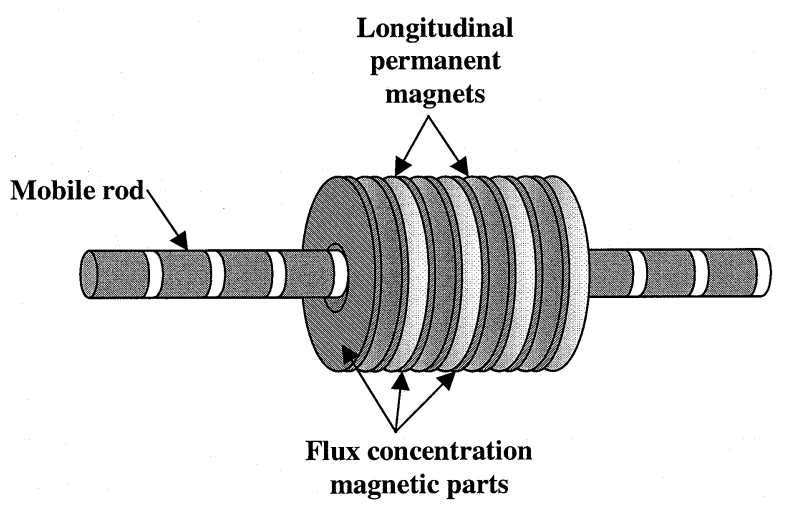

Fig. 11. One of the eight rods of the multi-rod actuator.

mobile parts are rods. Compared to the central rod actuator, the multi-rod actuators can reach greatest cumulative air-gap surface, which is better for high force density.

This cylindrical shape leads to the D-pattern, which features an axis of symmetry (Fig. 11). Permanent magnets are placed

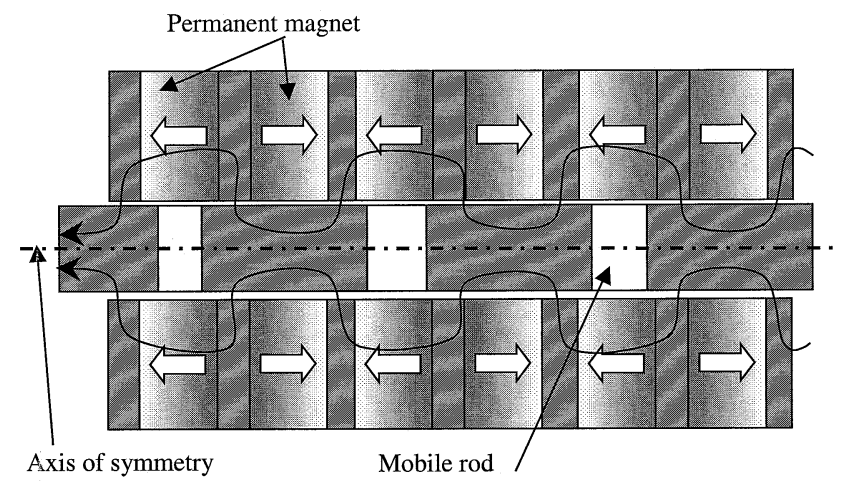

(a)

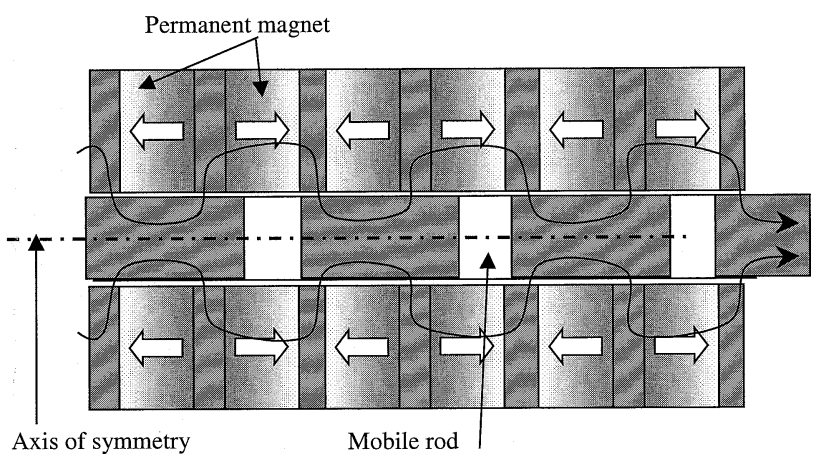

(b)

Fig. 12. (a) Positive alignment. (b) Negative alignment.

on the static part of the actuator. The mobile rods are composed of a succession of magnetic and nonmagnetic rings.

Fig. 12 shows the two alignment positions. The positive alignment position provides the maximum permanent-magnet flux, and the negative one, the minimum permanent-magnet flux.

The magnetic part of the rod is $4 \mathrm{~mm}$ in length, the nonmagnetic part is $1 \mathrm{~mm}$ in length, therefore, the step is $5 \mathrm{~mm}$ in length. The static permanent magnets are $1.5 \mathrm{~mm}$ in length. 


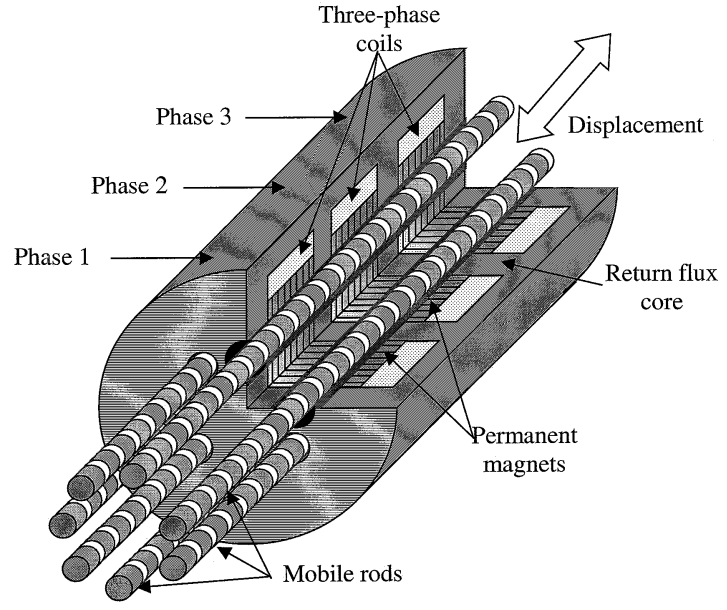

(a)

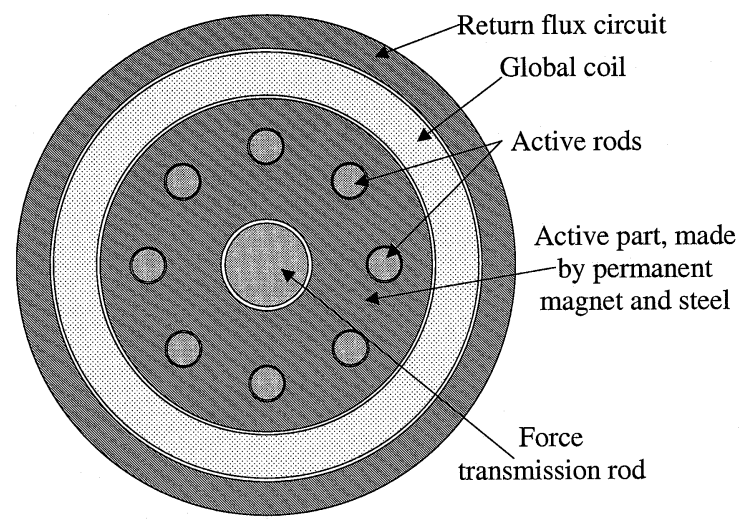

(b)

Fig. 13. (a) Operating principle of the multi-rod actuator. (b) Cross section of the actuator.

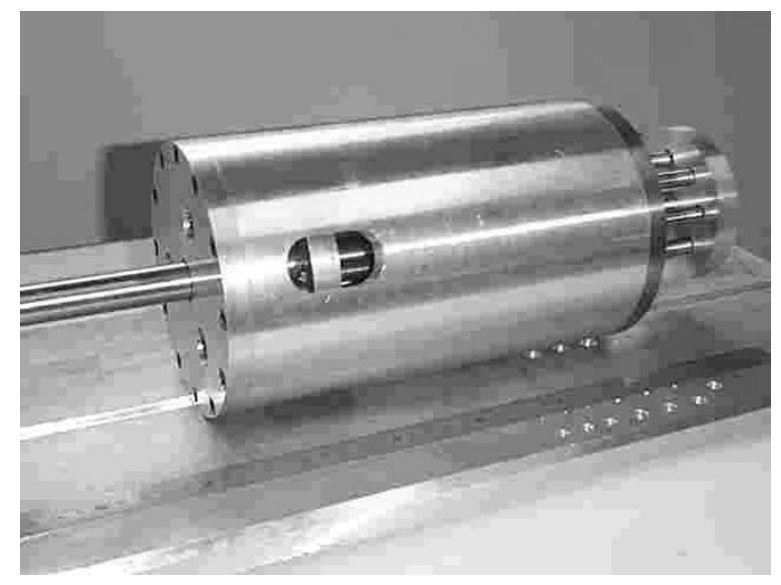

Fig. 14. Multi-rod prototype.

Three phases have been placed along the rods, as shown in Fig. 13. Fig. 14 shows the final structure.

\section{B. FEM Computation}

The significant mechanical complexity herein has prevented us from conducting a global simulation of the actuator. The same methodology as in Section II [6], [7], [8] has, thus, been applied, with only an elementary pattern being modeled by the FEM.

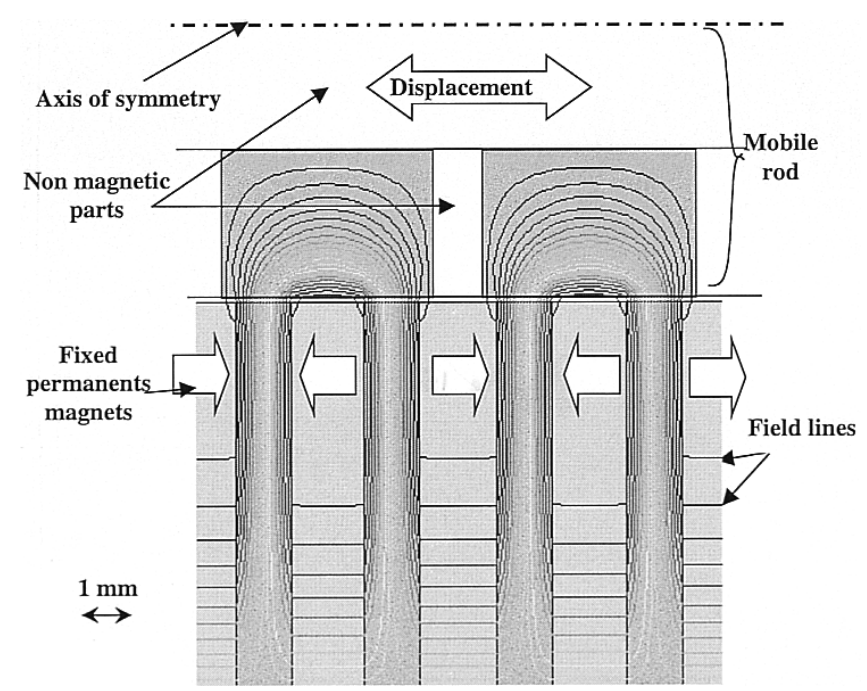

Fig. 15. Permanent-magnet flux in the positive alignment position of two elementary patterns.

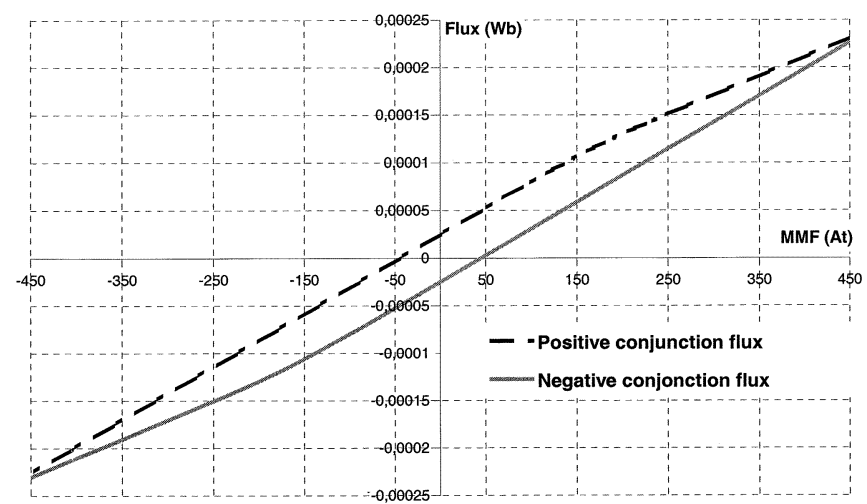

Fig. 16. Flux versus MMF: maximal and minimal curves of the pattern (in Wb/At).

First, the magnetic salience of the pattern was calculated. This result yielded an $L_{d} / L_{q}$ ratio of 0.984 . Hence, we may neglect the reluctance force in this pattern. Under these conditions, we decided to limit our study to just the alignment positions. Intermediate flux positions were then extrapolated between these two alignment positions. This interpolation is based on the fact that we only consider the first harmonic of the permanent-magnet flux. For a given current value, the actuator flux versus position is sinusoidal, with the maximal value reach of the positive alignment position and the minimum value for the negative alignment position.

Thanks to the axial symmetry of the pattern, a two-dimensional (2-D) FEM can be used. Fig. 15 displays the field map of the FEM simulation.

It should be noted that in employing such an approach, the flux return part of the actuator is considered to be perfect. The global coil is modeled as a boundary condition of the elementary pattern by an MMF.

These calculations give the maximal and minimal curves of the flux of each pattern as a function of the MMF being applied on each of them (see Fig. 16).

Using these results, the maximal flux MMF of the total actuator has been calculated. The actuator has eight rods in parallel, 


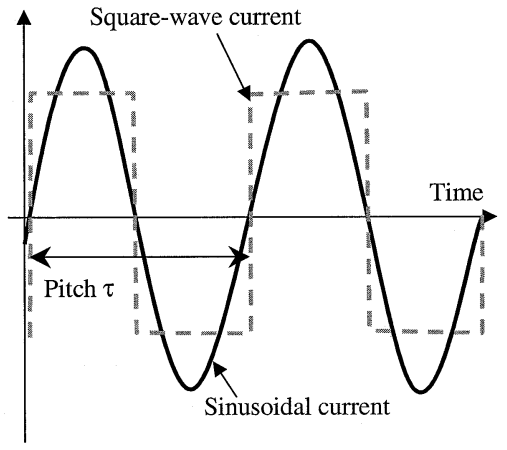

Fig. 17. Square-wave current and sinusoidal current for the same rms value.

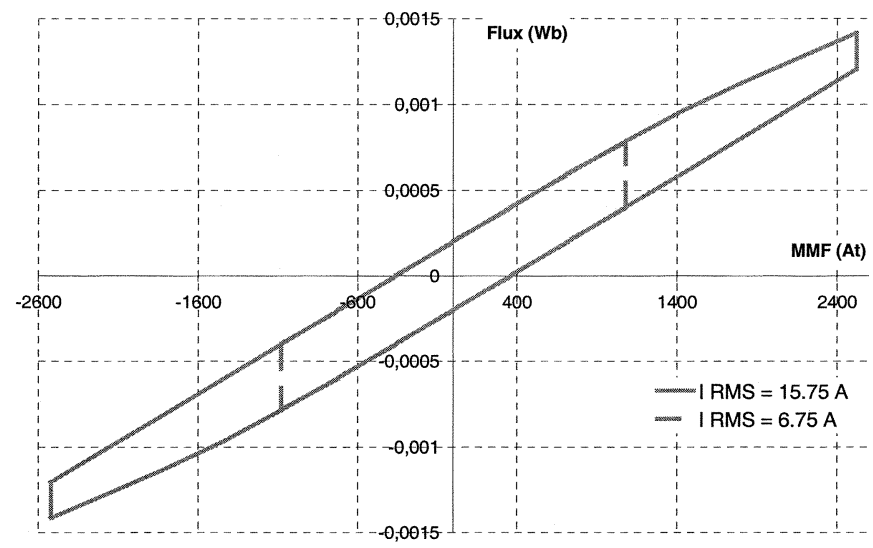

Fig. 18. Flux/MMF actuator loop with square-wave current (in Wb/At).

with each of the three phases being composed of eight elementary patterns in series. The flux and MMF of an elementary pattern are, hence, multiplied by eight in the actuator.

Two types of supplies have been considered herein: squarewave current and sinusoidal current (Fig. 17).

The square-wave current actuator force is simple to calculate by applying the conversion energy theory: for each stroke (pitch $\tau$ ) of the multi-rod motor, an energy $W_{m}$ is converted from an electrical form to an electromagnetic form. With square-wave currents, this energy can be expressed as (1):

$$
W_{m}=\oint_{n i} \varphi(n l) \cdot d n l=2 \cdot \int_{-n l_{\min }}^{n l_{\max }} \varphi(n l) \cdot d n l .
$$

The average electromagnetic force (EMF) $\bar{f}$ then equals (2)

$$
\bar{f}=\frac{W_{m}}{\tau} .
$$

The converted energy $W_{m}$ can be graphically calculated on the flux/magnetomotive loop (Fig. 18). For square-wave currents, Fig. 18 shows two loops for rms current values of $15.75 \mathrm{~A}$ $(2520 \mathrm{At})$ and $6.75 \mathrm{~A}(1080 \mathrm{At})$ :

For the sinusoidal current, (2) remains valid. Moreover, the converted energy $W_{m}$ is still represented on the flux/MMF loop. This loop has been drawn in considering that, for a given current, PM flux variation is sinusoidal. Fig. 19 presents the loop for rms currents of 15.9 and $8 \mathrm{~A}$, respectively.

By virtue of (2), we can then proceed with calculating the force as a function of rms current for two kinds of current as shown in Fig. 20.

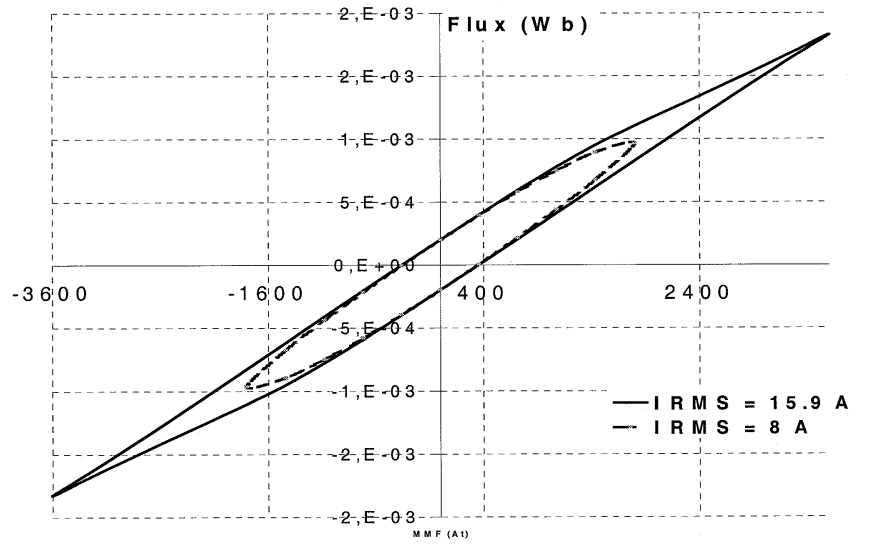

Fig. 19. Flux/MMF actuator loop with sinusoidal current (in Wb/At).

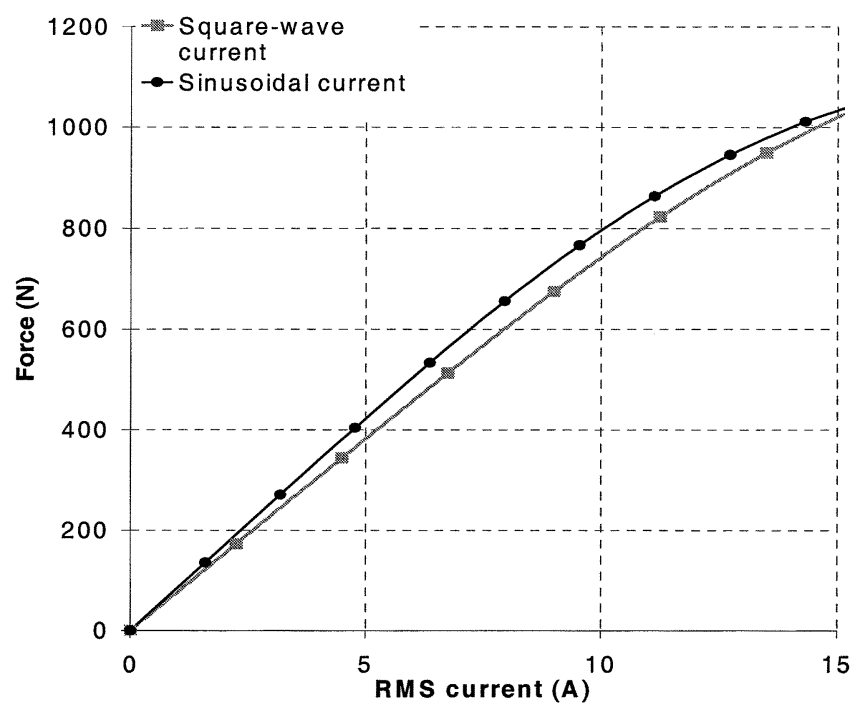

Fig. 20. Average force (in N) versus rms current (in A).

For the linear part of the curve (with sine flux waveform hypothesis), the ratio between the square-wave-fed actuator force and the sinusoidal force is (3)

$$
\frac{F_{\text {sin }}}{F_{\text {square }}}=\frac{\pi}{2 \cdot \sqrt{2}} \approx 1.11
$$

In contrast, saturation of magnetic parts limits the maximum conversion energy to a common value for both types of supply: the two curves have the same limit values.

\section{Normal Forces and Mechanical Guidance of the Multi-Rod Actuator}

One of the most difficult aspects in multi-air-gap actuators pertains to the mechanical guidance. In order to obtain a very high force-to-volume ratio, air-gap thickness must be minimized. Given that the maximum speed of the actuator is relatively low (less than $2.5 \mathrm{~m} \cdot \mathrm{s}^{-1}$ ), contact guidance is possible, yet the normal forces prove unstable. The solution of spread guidance has been adopted so as to minimize eccentricity and, thereby, the resulting normal forces (Fig. 21).

In order to avoid hyperstaticity, each mobile rod is mechanically independent. Guidance surfaces, composed of Teflon sheets, have been placed around each of the rods (Fig. 22). 


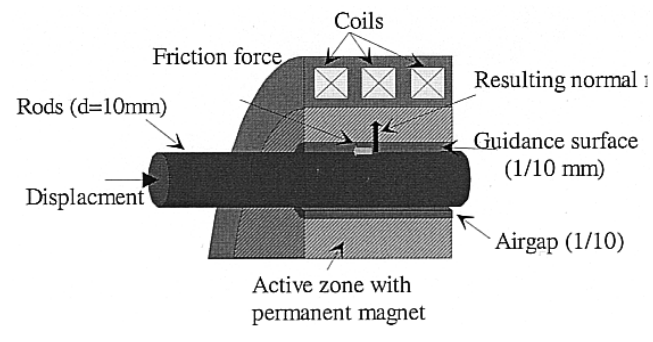

Fig. 21. Spread guidance operating principle.

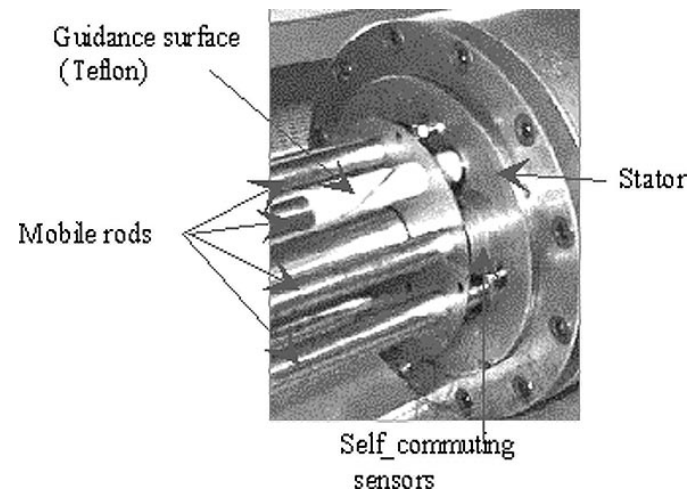

Fig. 22. Spread guidance setup.

\section{STATIC Forces AND DyNamic BeHAVIOR IN THE MULTI-ROD ACTUATOR}

\section{A. Static Force of the Multi-Rod Actuator}

Static force has been measured using the uncoupled method: coils are fed by direct current and a force sensor gives the maximum mechanical resistance of the actuator. Hence, we are able to measure the static EMF plus the friction force with the following method (Fig. 23).

The pertinent results are summed up in Fig. 24.

The friction force is estimated at $130 \mathrm{~N}$. This friction force is far more important that the cogging force, which is almost negligible. EMF is almost sinusoidal, and we can say that the force versus position in self-commuting condition will be constant.

Since the cogging force due to the permanent magnet is very low (less than $10 \mathrm{~N}$ ), this uncoupled test produces the force of the self-commuted actuator under generator conditions as a function of maximum current.

In order to derive the force as a function of rms current, the current scale would have to be modified.

The motor force is equal to the generator force minus twice the friction force.

All results are summed up in Fig. 25.

Given that the magnetic salience is very low, the optimal selfcommutation angle between the current and the EMF is close to $0^{\circ}$ (which means that no reluctance force is present).

We will now compare the low-speed EMF (without friction and magnetic losses) to the simulation results under sinusoidal current conditions (Fig. 26). Moreover, the EMF has already been measured; it can yield the slope of the force over the linear part of the curve.

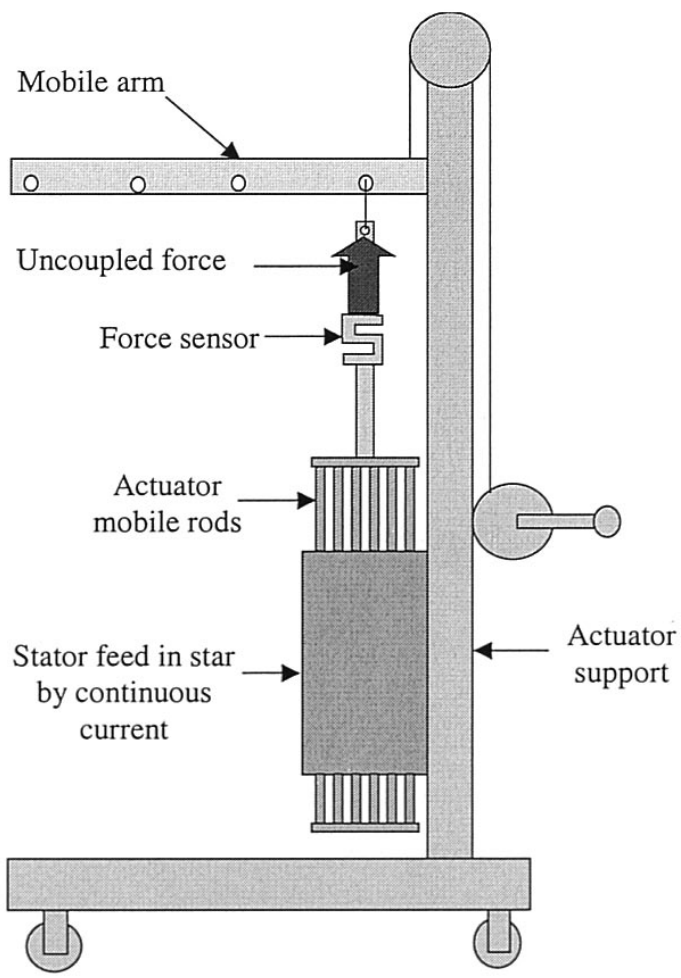

Fig. 23. Uncoupled test principle.

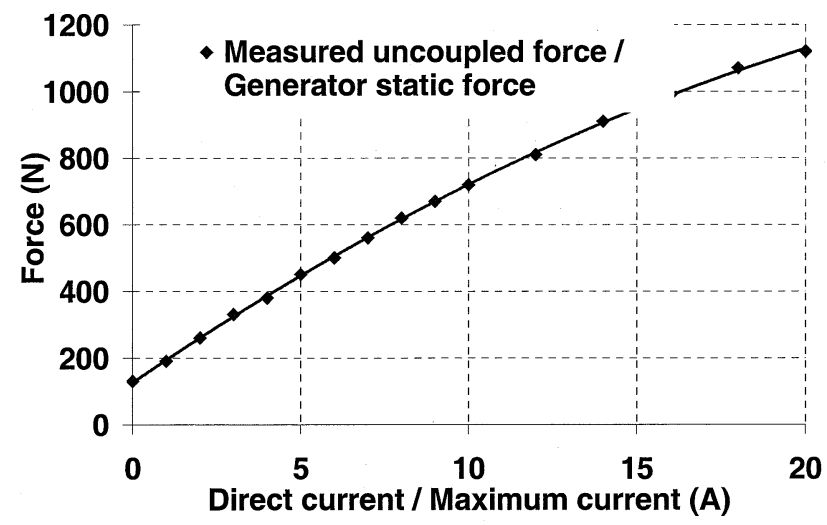

Fig. 24. Static force versus maximum current.

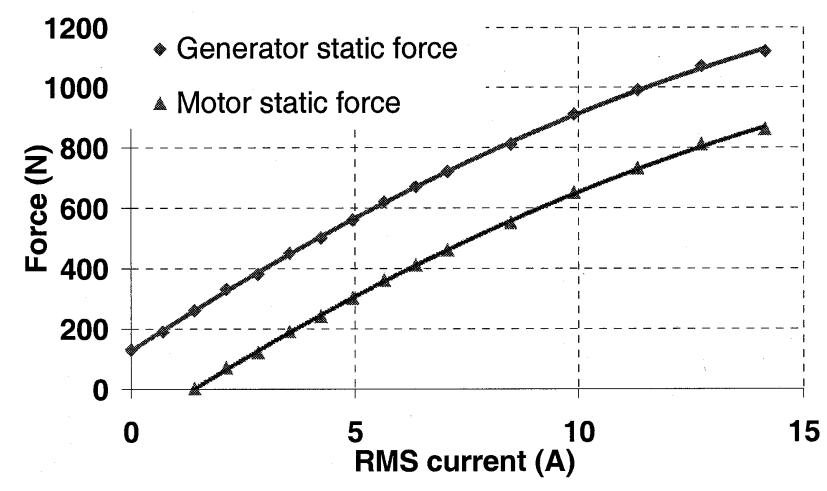

Fig. 25. Generator and motor static forces versus current.

We are able to state that the measurements show a very close fit with simulation values. Then, in our actuator, permanentmagnet flux waveform is effectively sinusoidal. 


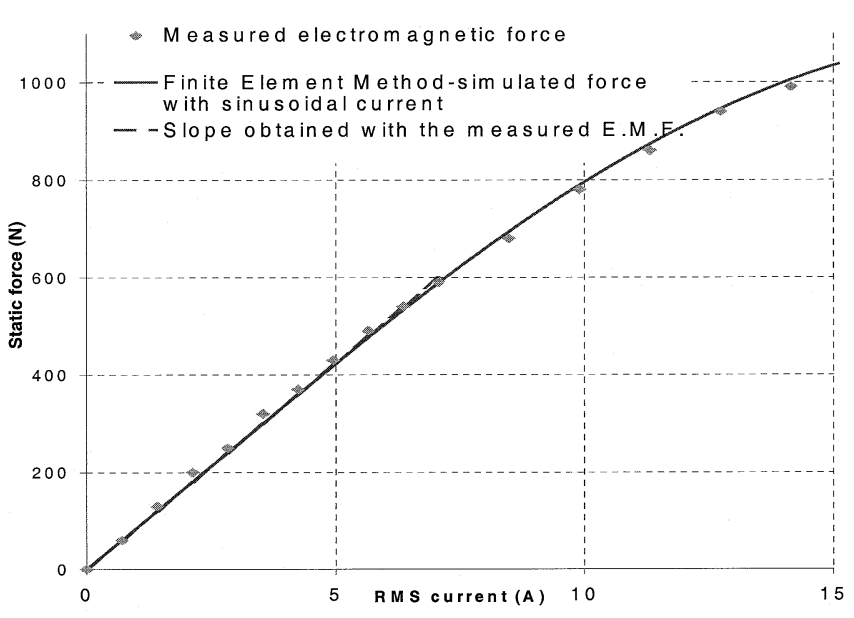

Fig. 26. Low-speed force comparison between static measurements and FEM simulation.

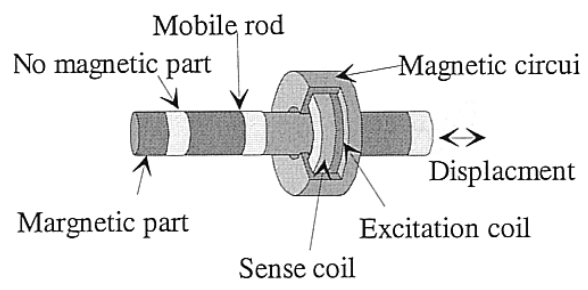

Fig. 27. Electromagnetic structure of the sensor.

The overall volume of this actuator is about $1 \mathrm{dm}^{3}$. Measurements indicate a maximum EMF of $1000 \mathrm{~N}$. We have therefore reached a force-to-volume ratio of $1000 \mathrm{~N} / \mathrm{dm}^{3}$, which is three times the value obtained for classical electromagnetic linear actuators $\left(\cong 300-400 \mathrm{~N} / \mathrm{dm}^{3}\right)$ [11].

We can conclude that this multi-rod actuator serves to validate the theoretical interest of multi-air-gap actuators for high force-to-volume ratios.

\section{B. Self-Commutation}

In order to self-commutate the actuator, integrated sensors have been developed that make use of the actuator rod [12] (Fig. 27); these sensors are designed to be automatically synchronous with the actuator position.

They have been optimized for maximum compaction (see Fig. 21).

One of the difficulties in self-commutation within such an actuator concerns the load. The mechanical time constant is very low and depends on the friction force. An adapted linear load is required to carry out self-commutation.

We have chosen a passive inertial load, as shown in Fig. 28. This mass is composed of 5-kg modular elements and has been placed on a linear motion guide. A car damper can be added, for example, to increase the load losses.

Self-commutation with a three-phase converter is currently being tested on this pure inertial load.

Fig. 19 shows the evolution of the flux in the actuator versus the current. We can see that the armature flux is rather important compared to the magnet flux. This means that the power factor of this actuator is rather poor at high speed. For the maximal rms current (15 A), we have a power factor of 0.1. This

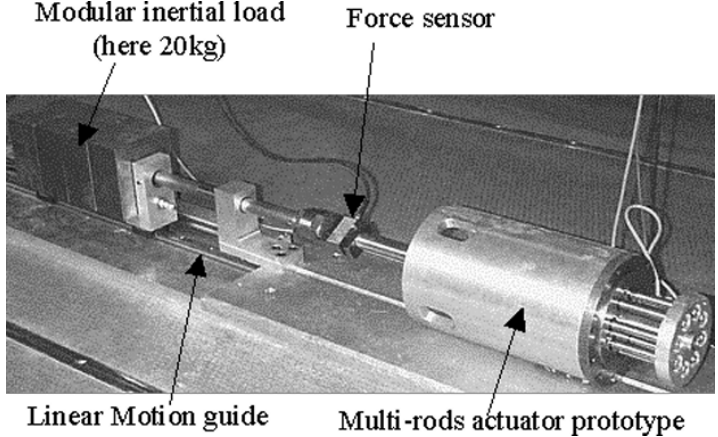

Fig. 28. Mechanical load of the multi-rod actuator prototype.

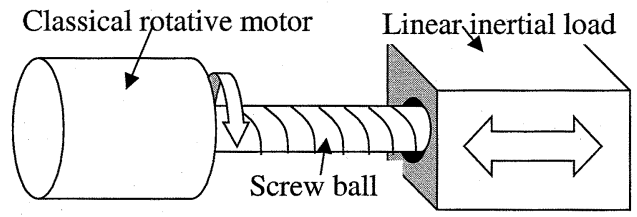

Fig. 29. Indirect linear actuator: "ball-screw" system principle.

is a common characteristic to high force density actuators (like transverse flux actuators). It will limit the use of such actuators to specials applications, where the price of the static converter could be accepted. However, at very low speed, mechanical power is often low in regard to copper losses and power factor becomes good.

From a production point of view, it appears that these actuators could be built at a reasonable price. Even though the mechanical pieces are numerous, they are easy to build. The linkage between those mechanical part could be made by composite material. Moreover, the global volume of permanent magnet is rather low compared to classical permanent magnet actuators with permanent magnets on the rods.

It remains true that, like all high-performance actuators, this will never be suited to very cheap applications.

\section{Scaling Effects for Wide Mechanical Bandpass Applications}

Wide mechanical bandpass linear applications have been introduced in numerous instances. The following can be cited for purposes of illustration:

- compensation of high-speed train vibrations;

- parallel robots for the high-speed manipulation of heavy loads;

- plane aileron drives.

This wide mechanical bandpass may be expressed relatively well by the maximum acceleration of the linear load. For the sake of simplicity, we will consider the load as a pure inertial load.

For linear movement, we can compare two kinds of solutions:

- classical "ball-screw" system (Fig. 29), in which a ball screw is placed between the rotative electrical actuator and the linear load;

- direct-drive solution, in which the linear electrical actuator is directly linked to the linear load.

For the "ball-screw" solution, a key parameter is the coefficient of conversion $k$ between the linear movement and the 
rotative movement $(k=\Omega / v)$. With a rotative actuator, the internal inertia is called $J$ and the nominal torque $T_{n}$. If the linear load were a pure inertial load of mass $M$, the linear acceleration would be (4)

$$
a=\frac{T_{n} \cdot k}{M+k^{2} . J} .
$$

The optimal value (acceleration maximization) of $k$ is (5)

$$
k_{o p t}=\sqrt{\frac{M}{J}} .
$$

With this value, the maximum acceleration becomes (6)

$$
a_{\max }=\frac{T_{n}}{2 \cdot \sqrt{J}} \frac{1}{\sqrt{M}} \text {. }
$$

The first part of this equation relates to the actuator: this value is directly linked to the size of the actuator. The scaling effect gives the evolution of torque and inertial load as a function of dimension $l^{*}$ [13].

For a thermal operating point, the nominal torque of the rotating permanent magnet motor is (7)

$$
\frac{T_{n}}{V} \propto l^{* 0.5} \cdot k_{e}^{0.5}
$$

where $k_{e}$ is the thermal coefficient (expressed in terms of $\left.\mathrm{W} \cdot \mathrm{m}^{-2} \cdot \mathrm{K}^{-1}\right)$.

The rotor inertia is $(8)$

$$
J \propto l^{* 5} .
$$

Hence, maximum acceleration is linked to the size of the actuator as follows:

$$
a_{o p t} \propto \frac{l^{* 3.5} \cdot k_{e}^{0.5}}{2 . l^{* 2.5}} \cdot \frac{1}{\sqrt{M}} \propto \frac{l^{*}}{\sqrt{M}} .
$$

For a direct-drive linear actuator, two kinds of actuator can be chosen:

- classical linear permanent magnet synchronous motors;

- multi-rod actuators.

For both solutions, the mobile part of the actuator is relatively light. For our prototype, the rods weigh around $1.3 \mathrm{~kg}$ and are, thus, negligible in comparison with the weight of the load (between $10-100 \mathrm{~kg}$ for our actuator). The acceleration is (10)

$$
a=\frac{F_{n}}{M}
$$

where the nominal force is linked to the size of the actuator.

Classical linear permanent-magnet synchronous motors display, under given thermal conditions, a constant force-to-volume ratio [9] (11)

$$
\frac{F_{n}}{V} \propto k_{e}^{0.5} .
$$

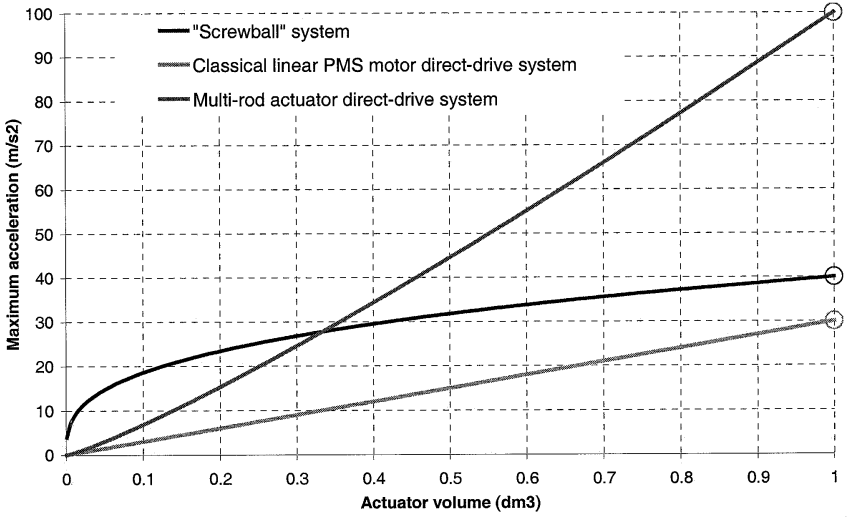

Fig. 30. Maximum acceleration for a linear inertial load of $10 \mathrm{~kg}$ versus actuator volume.

The maximum acceleration follows the scaling rule (12)

$$
a \propto \frac{l^{* 3}}{M}
$$

The same scaling effect can then be applied to the multi-rod actuator [9] (13)

$$
\frac{F_{n}}{V} \propto \frac{l^{* 0.5} \cdot k_{e}^{0.5}}{e}
$$

where $e$ is the air gap, linked to a mechanical technology. For our purposes, we will maintain a constant air gap. The maximum acceleration follows the scaling rule (14)

$$
a \propto \frac{l^{* 3.5}}{M} .
$$

For our multi-rod actuator, for instance, $l^{*}=0.1 \mathrm{~m}(V=$ $\left.1 \mathrm{dm}^{2}\right)$ :

$$
F_{n}=1000 \mathrm{~N} .
$$

For the same volume $\left(1 \mathrm{dm}^{3}\right)$, the force of a classical permanentmagnet synchronous motor is approximately

$$
F_{n}=300 \mathrm{~N} .
$$

Without taking the inertia and ball-screw volume into account, the best rotative actuator has the following values:

$$
\begin{aligned}
T_{n} & =3.3 \mathrm{Nm} \\
J & =0.17 \cdot 10^{-3} \mathrm{~kg} \cdot \mathrm{m}^{2} .
\end{aligned}
$$

The scaling effect provides two system comparisons: light load ( $M=10 \mathrm{~kg} ; k=242 \mathrm{rad} / \mathrm{m})$ (Fig. 30), and heavy load $(M=100 \mathrm{~kg} ; k=767 \mathrm{rad} / \mathrm{m})$ (Fig. 31).

In Figs. 29 and 30, the reference values have been represented by an empty circle. Comparing the ball-screw solution and the linear permanent-magnet motor solution, the first one is interesting for small motor volumes whereas the second one is interesting for greater actuators. 


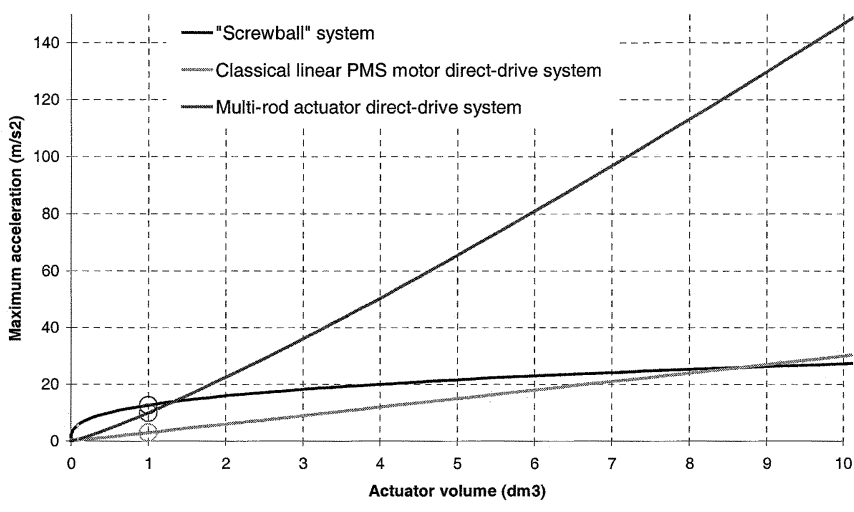

Fig. 31. Maximum acceleration for a linear inertial load of $100 \mathrm{~kg}$ versus actuator volume.

Moreover, it is apparent that direct-drive systems become worthwhile only once acceleration has reached high levels. This efficiency increases with heavier loads.

\section{CONCLUSION}

This paper has focused on a new multi-air-gap synchronous actuator dedicated to high mechanical bandpass applications.

The FEM has yielded results very comparable to measurements with the multi-rod prototype under sinusoidal current conditions, despite a very complex magnetic structure.

The performance of this actuator is very attractive (around $1000 \mathrm{~N} / \mathrm{dm}^{3}$ with a force of $1000 \mathrm{~N}$ ) and proves the interest in the multi-air-gap technology.

Mechanical guidance problems have been resolved thanks to an original spread guidance solution. We are now working toward minimizing the normal force and searching for the best compromise between tangential and normal forces.

Furthermore, the theoretical advantages of direct-drive solutions for wide mechanical bandpass applications have been demonstrated.

Typical applications of these kinds of actuators necessitate high force densities and high mechanical bandpass. We can quote compensation of high-speed train vibrations, parallel robots for the high-speed manipulation of heavy loads, or plane aileron drives.

\section{REFERENCES}

[1] B. Multon and J. Bonal, "Electromechanical direct drives: Variety, constraints and solutions" (in French), Elect. Eng. Rev. (REE), vol. 10, pp. 67-80, Nov. 1999.

[2] U. S. Deshpande et al., "High-force density linear switched reluctance machine," IEEE Trans. Ind. Applicat., vol. 31, pp. 345-352, Mar./Apr. 1995.

[3] C. Rioux, "Théorie générale comparative des machines électriques établie à partir des équations du champ électromagnétique," Rev. Gen. Elect., vol. 79, no. 5, pp. 415-421, May 1970.

[4] D. Matt, R. Goyet, J. Lucidarme, and C. Rioux, "Longitudinal field multi-airgap linear reluctance actuator," Elect. Mach. Power Syst., vol. 13, pp. 299-313, 1987.

[5] P. E. Cavarec et al., "Force density improvements from increasing the number of airgap surfaces in synchronous linear actuators," Proc. IEE_Elect. Power Applicat., vol. 150, no. 1, p. 106, Jan. 2003.
[6] P. E. Cavarec et al., "Advantage of increasing the number of airgap surfaces in synchronous linear actuators," in Proc. Electromotion 2001, Bologna, Italy, June 19-20, 2001, pp. 251-256.

[7] D. Staton, W. Soong, and T. J. Miller, "Unified theory of torque production in switched reluctance and synchronous reluctance motors," IEEE Trans. Ind. Applicat., vol. 31, pp. 329-337, Mar./Apr. 1995.

[8] D. Staton, R. P. Deadhar, W. L. Soong, and T. J. Miller, "Torque prediction using the flux-MMF diagram in AC, DC and relucstance motors," IEEE Trans. Ind. Applicat., vol. 32, pp. 180-188, Jan./Feb. 1996.

[9] H. Ben Ahmed, L. Prévond, B. Multon, B. Salamand, and J. Lucidarme, "Special synchronous linear actuators: Structures and performances," Rev. Electromotion, no. 5, pp. 93-101, 1998.

[10] P. F. Desesquelles, J. Lucidarme, and H. Ben Ahmed, "Theoretical and experimental results on multi-airgap axial synchronous machines with permanent magnets," presented at the ICEM'90, Cambridge, MA, Aug. 1990.

[11] N. Bianchi et al., "Tubular linear permanent magnet motors: An overall comparison," presented at the IEEE-IAS Annu. Meeting, Pittsburgh, Oct. 13-18, 2002.

[12] P. E. Cavarec et al., "Integrated reluctance position sensor for the selfcommutation of a hybrid linear electric actuator," in Proc. EPE 2001, Graz, Austria, Aug. 2001, CD-ROM.

[13] M. Jufer et al., "Laws governing the size reduction of electromechanical transducers with applications to step motors," Dept. Elect. Eng., Univ. Illinois, Urbana-Champaign, IL, 1974.

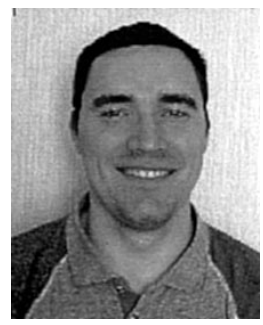

Pierre-Emmanuel Cavarec was born in Brest, France, in 1975. He received the engineer diploma and the Agrégation from Supelec (ESE), Gif sur Yvette, France, in 1998 and 1999, respectively, and the $\mathrm{Ph} . \mathrm{D}$. degree from the Ecole Normale Supérieure de Cachan-Brittany, Bruz, France, in 2002.

He is currently with Harmonic Design Inc., Somfy International, Poway, CA. His main research interest is unconventional linear motors.

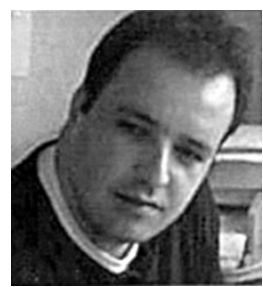

Hamid Ben Ahmed received the Engineer, D.E.A., and Ph.D. degrees from the University of Paris 6, Paris, France, in 1988, 1989, and 1994, respectively.

From 1994 to 1997, he was a Research Associate in the SATIE Laboratory, Ecole Normale Supérieure de Cachan-Brittany, Bruz, France, where, since 1997, he has been an Assistant Professor in the Mechatronics Department. His research interests include the design, modeling, and optimization of novel topologies of electromagnetic actuators and generators, and the optimization of renewable energy systems.

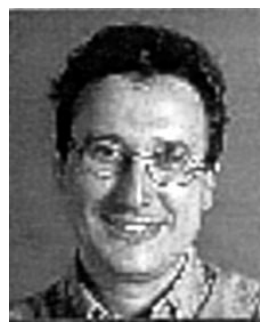

Bernard Multon (M'97) was born in 1960. He received the M.S. degree in electrical engineering and the Agrégation from the Ecole Normale Supérieure de Cachan, Cachan, France, in 1981 and 1982, respectively, and the Ph.D. degree from the University of Paris 6, Paris, France, in 1985.

From 1988 and 1995, he was an Professor in the Electrical Engineering Department, Ecole Normale Supérieure de Cachan, and Leader of the Research Special Actuator Design Group, Laboratoire Electricité, Signaux et Robotique (LESiR, ESA CNRS 8029). Since 1995, he has been a Full Professor in the Ecole Normale Supérieure de Cachan-Brittany, Bruz, France. He is head of the Mechatronics Department and of the Actuators-Energy Research Group of the Systems and Applications of Technologies in Information and Energy (SATIE) with CNRS. His research activities essentially concern new actuators (reluctance and permanent magnet, linear and rotating) study and new energy production systems (renewable and human power). 